\title{
Family-based study of association between MAFB gene polymorphisms and NSCL/P among Western Han Chinese population
}

\author{
Bihe Zhang ${ }^{1, B, D}$, Shijun Duan ${ }^{1, A-F}$, Jiayu Shi ${ }^{2, B, C}$, Shuyuan Jiang ${ }^{1, B, C}$, Fan Feng ${ }^{3, B, C}$, Bing Shi, ${ }^{1, A, E}$, Jia Zhonglin ${ }^{1, A, C, E, F}$ \\ 1 State Key Laboratory of Oral Disease, West China College of Stomatology, Sichuan University, Chengdu, China \\ 2 Division of Growth and Development and Section of Orthodontics, School of Dentistry, University of California, Los Angeles, USA \\ ${ }^{3}$ Air Force Dujiangyan Aviation Medicine Evaluation and Training Center, Chengdu, China \\ A - research concept and design; $B$ - collection and/or assembly of data; $C$ - data analysis and interpretation; \\ $D$ - writing the article; $E$ - critical revision of the article; $F$ - final approval of the article
}

Address for correspondence

Zhonglin Jia

E-mail:zhonglinjia@sina.com

Funding sources

The National Science Funds of China

(No. 81600849, No. 81271118) and Scientific

Research Funds for Young Teachers of Sichuan

University (No. 0040305505088)

Conflict of interest

None declared

Received on March 14, 2017

Reviewed on May 25, 2017

Accepted on June 3, 2017

\begin{abstract}
Background. Non-syndromic cleft lip with or without cleft palate (NSCL/P) are the most common human congenital birth defects with a complex etiology. MAFB has been reported as a candidate gene involved in the pathogenesis of NSCL/P from genome-wide association study (GWAS) findings, and no replication studies have been performed in Western Han Chinese.

Objectives. The aim of this study was to investigate the associations of MAFB among NSCL/P trios in Western Han Chinese.

Material and methods. We selected 6 single nucleotide polymorphisms (SNPs) (rs6072081, rs6065259, rs17820943, rs13041247, rs11698025 and rs6102085) near MAFB based on previous GWAS findings and recruited 298 case-parents trios with NSCL/P from Western Han Chinese population, while genotypes were done by SNPscan technology.

Results. Strong evidence of an association was found at rs 17820943 ( $p=0.0023$; odds ratio $-0 R_{\text {transmission }}$ $=0.7$ and 95\% confidence interval [CI]: 0.55-0.88) and rs13041247 ( $p=0.0023 ; 0 R_{\text {transmission }}=0.7$ and 95\% Cl: 0.55-0.88) among NSCL/P; genotypic transmission-disequilibrium test (TDT) analysis further confirmed this. C/C homozygote at rs $17820943(z=3.44$ and $p=0.00058)$ and T/T homozygote at rs13041247 $(z=3.14$ and $p=0.0017)$ were over-transmitted among NSCL/P, which indicated they could increase the risk of having an affected baby. Sliding window haplotype analysis showed that haplotypes consisting of C allele at rs17820943 and T allele at rs13041247 were still over-transmitted among NSCL/P (lowest $p=0.0021)$.
\end{abstract}

Conclusions. This study further confirmed that the targeted SNPs at MAFB were associated with NSCL/P trios from Western Han Chinese population, which provides more scientific evidence for the future research and genetic counseling.

Key words: single nucleotide polymorphism, transmission disequilibrium test, MAFB, haplotype analysis, non-syndromic cleft lip with or without cleft palate

DOI

10.17219/acem/74388

Copyright

Copyright by Author(s)

This is an article distributed under the terms of the

Creative Commons Attribution Non-Commercial License

(http://creativecommons.org/licenses/by-nc-nd/4.0/) 


\section{Introduction}

Cleft lip with or without cleft palate (CL/P) are the most common congenital craniofacial birth defects, which can be categorized as an isolated anomaly (identified non-syndromic CL/P - NSCL/P) or as a part of multiple congenital anomalies (identified syndromic CL/P - SCL/P). The cases of NSCL/P are more prevalent (approx. at $70 \%$ of all cleft cases), with the worldwide occurrence incidence ranging from $1 / 500$ to $1 / 2500 .{ }^{1}$ Since CL/P influences the structure of the face and oral cavity, the affected children need to confront a wide range of difficulties, including feeding, speech, hearing and dental defects, as well as appearance and psychosocial problems, which can induce long-lasting health and financial burdens. ${ }^{2}$ Generally, the prevalence of NSCL/P was 1.42 per 1000 in Chinese newborns, presenting a high frequency among countries worldwide, which is an important public health problem in China. ${ }^{3}$ Actually, some epidemiological and biological data suggested NSCL/P might be divided into non-syndromic cleft lip only (NSCLO) and non-syndromic cleft lip with cleft palate (NSCLP) which should be analyzed separately and are considered to have different genetic etiologies and embryo origins. ${ }^{4,5}$ The origin of cleft lip was seen as a malformation of the primary palate only, while cleft lip with cleft palate involved both the primary and secondary palates. ${ }^{4}$ There were obvious discordances regarding the recurrence risks and transmission patterns between these two subgroups. ${ }^{5}$ Therefore, to explore the further possible susceptibility genes, the 2 subgroups should be analyzed separately.

NSCL/P were genetically complex disorders, which included the interaction of multiple genetic and environmental risk factors. The search for susceptibility genes of NSCL/P was undertaken using numerous approaches, including animal experiments, candidate genes, linkage analysis studies, genomic rearrangements; genome-wide association studies (GWAS), and direct sequencing. However, the results were still unsatisfactory. ${ }^{2,6}$ As a useful method for unraveling the genetic traits of common human diseases, GWAS quickly became the new standard in genetic analysis at the genome level and it identified multiple strong association susceptibility genes. ${ }^{7,8}$ So far, the GWAS of NSCL/P had identified more than 15 susceptible loci, which provided more insights into the genetic etiology of NSCL/P. ${ }^{9-15}$ However, the pathogenesis of NSCL/P involving these loci was still unclear. Although the advantages of GWAS were obvious, some potential limitations of this method might influence the results, or miss disease heritability, even leading to a false positive result. ${ }^{16,17}$ Population-based association studies were always designed and performed in the majority of GWAS. ${ }^{7,18}$ Though the difficulty of sample collection was avoided, the presence of undetected population substructure might be missed or affect the signal of significant association.,18 Subsequent family-based association studies (such as case-parents trios study), focusing on limited genetic markers after GWAS findings, were more efficient in correcting the population substructure and more powerful in revealing cryptic relatedness of samples. ${ }^{7,16}$

Up to now, the largest GWAS about non-syndromic orofacial clefts was conducted by Beaty et al., who were the first to report that single nucleotide polymorphisms (SNPs) in or near MAFB (v-maf musculoaponeurotic fibrosarcoma oncogene homolog B; located in 20q12) are associated with NSCL/P, with the most significance coming from an analysis of families with Asian ancestry population, including samples from Western Han Chinese collected in our hospital. ${ }^{12} M A F B$, encoding a basic leucine zipper (bZIP) transcription factor, had been found to play a significant role in the development of brain, kidney, lens, retina, pancreatic islet cells, and the hematopoietic system. ${ }^{19}$ However, according to findings by Beaty et al., the expressions of $M A F B$ mRNA and protein had also been demonstrated in both craniofacial neuroectoderm and neural-crest derived from mesoderm between embryonic day 13.5 and 14.5, which suggested that the gene might associate with NSCL/P closely. ${ }^{12}$ Then, interactions between $M A F B$ and other genes were detected in both European and Asian CL/P populations through gene-gene interaction analysis. ${ }^{17}$ Subsequently, several validation studies had been done in the United States, Brazil, Colombia, and China. ${ }^{20-25}$ Nonetheless, the results of those studies were inconsistent with each other, as only limited populations were validated in some studies or different research methods were adopted. Then, based on Chinese Han population, Sun et al. conducted a 3-stage case-control GWAS and still found some SNPs (locating in downstream of $M A F B$ ) at $20 \mathrm{q} 12$ involving in orofacial development. The majority of samples in this study also came from the Western Han Chinese population treated in our hospital (West China Hospital of Stomatology, Sichuan University, Chengdu, China). ${ }^{14}$

Although the results of these studies were encouraging, only a part of Chinese population was included in the validation research and associations with certain subgroup of NSCL/P were not fully elucidated by Beaty et al. and Sun et al. ${ }^{12,14}$ Meanwhile, the Western Han Chinese ancestry population also contributed to the findings of Beaty et al. and Sun et al. ${ }^{12,14}$ To know if the GWAS signals were associated with cleft palate (CP), we previously recruited 144 case-parents trios with $\mathrm{CP}$, and tested the associations with 38 SNPs, which included 6 SNPs at MAFB (rs6072081, rs6065259, rs17820943, rs13041247, rs11698025 and rs6102085). A single SNP analysis did not show any significant relevance between $M A F B$ and CP; however, interactions between 2 SNPs show that rs6072081 significantly interacted with rs6102085, which indicated that these 2 SNPs may act in the same pathway in the etiology of CP. ${ }^{26}$

Thus, in this study, we used another set of samples with NSCL/P trios different from the ones used in the 2 previous GWASs (Beaty et al. and Sun et al.) and conducted 
a validation study with 6 SNPs at MAFB: (rs6072081, rs6065259, rs17820943, rs13041247, rs11698025 and rs6102085) to investigate whether these loci were associated with NSCLO, NSCLP and NSCL/P in Western Han Chinese population. ${ }^{12,14}$

\section{Material and methods}

\section{Ethics statement}

We obtained a written informed consent from all participants or legal guardians of the affected children younger than 16 years before they were enrolled in the study. The study protocols were reviewed and approved by the Hospital Ethics Committee (HEC) of West China Hospital of Stomatology, Sichuan University, Chengdu, China.

\section{Samples description}

Our samples included 298 case-parent trios, who were recruited between 2008 and 2013 from the Cleft Lip and Palate Surgery Department of West China Stomatology College, Sichuan University, Chengdu, China. All probands were checked by professional maxillofacial doctors, with congenital deformities diagnosed as non-syndromic and major developmental delays excluded. All participants were identified as Western Han Chinese according to selfidentification. The gender and types of clefts is shown in Table 1.

Table 1. Types of non-syndromic cleft lip with or without cleft palate

\begin{tabular}{|l|c|c|c|c|}
\hline Phenotype & Male & Female & Unknown sex & Total \\
\hline NSCLO & 70 & 57 & 0 & 127 \\
\hline NSCLP & 111 & 55 & 5 & 171 \\
\hline NSCL/P & 181 & 112 & 5 & 298 \\
\hline
\end{tabular}

NSCLO - non-syndromic cleft lip only; NSCLP - non-syndromic cleft lip with cleft palate; NSCL/P - non-syndromic cleft lip with or without cleft palate (NSCLO and NSCLP); NSCPO - non-syndromic cleft palate only.

\section{SNPs selection and genotyping}

Based on the findings of Beaty et al., we chose 6 SNPs (rs6072081, rs6065259, rs17820943, rs13041247, rs11698025 and rs6102085) with significant p-values near $M A F B$, with minor allele frequency (MAF) $>0.30 .{ }^{12}$ Primary information of these SNPs is shown in Table 2.

Venous blood samples were drawn from all participants after being recruited in the study. Genomic DNA was extracted by using the protein precipitation method. ${ }^{27}$ All the experiments of genotyping were done by the Genesky Biopharm Technology Company, using SNPscan technology.

\section{Statistical analysis}

For each SNP, we checked for deviations by performing the Hardy-Weinberg equilibrium (HWE) test and calculated the MAF among the unaffected parents. Pairwise linkage disequilibrium (LD) was calculated as D' and $\mathrm{r}^{2}$ for all SNPs to identify LD blocks by the Haploview program (Cambridge, USA). The transmitted target alleles and genotypes from heterozygous parents to the affected child were evaluated by the transmission-disequilibrium test (TDT) analysis, and the Sliding window haplotype transmission disequilibrium analysis was conducted by familybased association test (FBAT) program (Cambridge, USA). We used a Bonferroni correction for the 18 tests to determine a threshold for formal significance of $\mathrm{p}=0.0028$.

\section{Results}

\section{Characteristic information of the study population}

Based on clinical manifestations, the selected NSCL/P samples were divided into 2 subgroups, 127 NSCLO cases (42.6\%) and 171 NSCLP cases (57.4\%), respectively. The observed genotype frequencies of NSCL/P were in agreement with the Hardy-Weinberg equilibrium ( $>$ > 0.01) (Table 2), indicating favorable genetic homogeneity within the study population.

\section{Allelic and genotypic TDT analysis}

Allelic TDT analysis was assessed on case-parents trios with the transmission of minor alleles from heterozygous informative parents to affected child. The results

Table 2. The minor allele frequency (MAF) and Hardy-Weinberg equilibrium results of single-nucleotide polymorphisms (SNPs)

\begin{tabular}{|c|c|c|c|c|c|c|c|c|c|c|}
\hline \multirow{2}{*}{ SNP } & \multirow{2}{*}{$\mathrm{BP}$} & \multirow{2}{*}{ SNP function } & \multirow{2}{*}{$\mathrm{A} 1$} & \multirow{2}{*}{$\mathrm{A} 2$} & \multicolumn{2}{|c|}{ NSCLO } & \multicolumn{2}{|c|}{ NSCLP } & \multicolumn{2}{|c|}{ NSCL/P } \\
\hline & & & & & MAF (\%) & HWpval & MAF (\%) & HWpval & MAF (\%) & HWpval \\
\hline rs6072081 & 39261054 & intergenic & G & $A$ & 40.29 & 1.00 & 41.0 & 0.03 & 40.59 & 0.15 \\
\hline rs6065259 & 39261979 & intergenic & A & G & 38.17 & 1.00 & 37.9 & 0.23 & 38.05 & 0.48 \\
\hline rs17820943 & 39268516 & intergenic & $\mathrm{T}$ & C & 40.14 & 1.00 & 40.4 & 0.10 & 40.26 & 0.24 \\
\hline rs13041247 & 39269074 & intergenic & C & $\mathrm{T}$ & 40.14 & 1.00 & 40.4 & 0.10 & 40.26 & 0.24 \\
\hline rs11698025 & 39274083 & intergenic & A & G & 31.20 & 1.00 & 33.5 & 0.58 & 32.20 & 0.71 \\
\hline rs6102085 & 39281629 & intergenic & A & $\mathrm{G}$ & 42.33 & 1.00 & 45.2 & 0.71 & 43.57 & 0.80 \\
\hline
\end{tabular}

NSCLO - non-syndromic cleft lip only; NSCLP - non-syndromic cleft lip with cleft palate; NSCL/P -non-syndromic cleft lip with or without cleft palate (NSCLO and NSCLP); HWpval - p-values of the Hardy-Weinberg equilibrium test. 
showed that minor allele T rs17820943 $(\mathrm{p}=0.0023$; odds ratio $-\mathrm{OR}_{\text {transmission }}=0.7$ and $95 \%$ confidence interval [CI]: 0.55-0.88) and allele $\mathrm{C}$ at rs113041247 ( $\mathrm{p}=0.0023$; $\mathrm{OR}_{\text {transmission }}=0.7$ and $95 \% \mathrm{CI}: 0.55-0.88$ ) was significantly under-transmitted from unaffected parents to probands among NSCL/P, which indicated that these alleles were protective for NSCL/P (Table 3).

Genotypic TDT was used to assess the genotype distribution comparison. The results showed significant overtransmission of $\mathrm{C} / \mathrm{C}$ homozygote at rs17820943 $(\mathrm{z}=3.44$ and $\mathrm{p}=0.00058), \mathrm{T} / \mathrm{T}$ homozygote at $\mathrm{rs} 13041247(\mathrm{z}=3.14$ and $\mathrm{p}=0.0017)$ and $\mathrm{G} / \mathrm{G}$ homozygote at rs11698025 $(\mathrm{z}=3.21$ and $\mathrm{p}=0.0013)$ from unaffected parents to probands among NSCL/P. Meanwhile, these 2 SNP markers were over-transmitted from unaffected parents to probands among NSCLP. Then, we observed A/A homozygote at rs6072081 ( $\mathrm{z}=3.051$ and $\mathrm{p}=0.0023)$ and G/G homozygote at $\operatorname{rs} 6065259(\mathrm{z}=3.38$ and $\mathrm{p}=0.00073)$ were also overtransmitted from unaffected parents to probands among NSCL/P (Table 4). However, no significant evidence of association was also identified in both allelic and genotypic TDT analysis for NSCLO.

Table 3. Allelic transmission-disequilibrium test (TDT) results for single nucleotide polymorphisms (SNPS) at MAFB

\begin{tabular}{|c|c|c|c|c|c|c|c|c|c|c|}
\hline \multirow{2}{*}{ SNP } & \multirow{2}{*}{ A1 } & \multicolumn{3}{|c|}{ NSCLO } & \multicolumn{3}{|c|}{ NSCLP } & \multicolumn{3}{|c|}{$\mathrm{NSCL} / \mathrm{P}$} \\
\hline & & $\mathrm{T} / \mathrm{U}$ & OR $(95 \% \mathrm{Cl})$ & $\mathrm{p}$-value & $\mathrm{T} / \mathrm{U}$ & OR $(95 \% \mathrm{Cl})$ & $\mathrm{p}$-value & $\mathrm{T} / \mathrm{U}$ & OR $(95 \% \mathrm{Cl})$ & $\mathrm{p}$-value \\
\hline rs6072081 & G & $62 / 75$ & $0.83(0.59-1.16)$ & 0.27 & $58 / 94$ & $0.62(0.44-0.86)$ & 0.0035 & $120 / 169$ & $0.71(0.56-0.9)$ & 0.0039 \\
\hline rs6065259 & $A$ & $57 / 62$ & $0.92(0.64-1.32)$ & 0.65 & $53 / 89$ & $0.6(0.42-0.84)$ & 0.0025 & 110/151 & $0.73(0.57-0.93)$ & 0.011 \\
\hline rs17820943 & $\mathrm{T}$ & $56 / 76$ & $0.74(0.52-1.04)$ & 0.08 & $63 / 95$ & $0.66(0.48-0.91)$ & 0.011 & $119 / 171$ & $0.7(0.55-0.88)$ & 0.0023 \\
\hline rs13041247 & C & $56 / 76$ & $0.74(0.52-1.04)$ & 0.08 & $63 / 95$ & $0.66(0.48-0.91)$ & 0.011 & $119 / 171$ & $0.7(0.55-0.88)$ & 0.0023 \\
\hline rs11698025 & A & $50 / 62$ & $0.81(0.56-1.17)$ & 0.26 & $51 / 83$ & $0.61(0.43-0.87)$ & 0.0057 & $101 / 145$ & $0.7(0.54-0.9)$ & 0.005 \\
\hline rs6102085 & $A$ & $65 / 58$ & $1.12(0.79-1.6)$ & 0.53 & $62 / 85$ & $0.73(0.53-1.01)$ & 0.06 & $127 / 143$ & $0.89(0.7-1.13)$ & 0.33 \\
\hline
\end{tabular}

NSCLO - non-syndromic cleft lip only; NSCLP - non-syndromic cleft lip with cleft palate; NSCL/P - non-syndromic cleft lip with or without cleft palate (NSCLO and NSCLP); A1 - minor allele; OR - odds ratio; 95\% Cl - 95\% confidence interval; bold characters indicate items with p-value less than 0.0028 (Bonferroni correction threshold p-value).

Table 4. Genotypic transmission disequilibrium test (TDT) results for single nucleotide polymorphisms (SNPS) on MAFB from family-based association test (FBAT)

\begin{tabular}{|c|c|c|c|c|c|c|c|c|c|c|c|c|c|}
\hline \multirow{2}{*}{ SNP } & \multirow{2}{*}{ Genotype } & \multicolumn{4}{|c|}{ NSCLO } & \multicolumn{4}{|c|}{ NSCLP } & \multicolumn{4}{|c|}{ NSCL/P } \\
\hline & & afreq & fam\# & z & $p$-value & afreq & fam\# & z & p-value & afreq & fam\# & z & p-value \\
\hline \multirow{3}{*}{ rs6072081 } & $\mathrm{G} / \mathrm{G}$ & 0.13 & 57 & -0.67 & 0.51 & 0.15 & 63 & -1.084 & 0.28 & 0.14 & 121 & -1.34 & 0.18 \\
\hline & $\mathrm{G} / \mathrm{A}$ & 0.56 & 95 & -0.72 & 0.47 & 0.49 & 125 & -1.7 & 0.089 & 0.52 & 221 & -1.68 & 0.093 \\
\hline & $\mathrm{A} / \mathrm{A}$ & 0.31 & 83 & 1.36 & 0.17 & 0.36 & 96 & 2.89 & 0.0039 & 0.34 & 179 & 3.051 & 0.0023 \\
\hline \multirow{3}{*}{ rs6065259 } & $A / A$ & 0.11 & 44 & -0.17 & 0.87 & 0.14 & 57 & -0.43 & 0.67 & 0.13 & 101 & -0.44 & 0.66 \\
\hline & $A / G$ & 0.51 & 88 & -0.85 & 0.39 & 0.47 & 118 & -2.76 & 0.0058 & 0.49 & 206 & -2.65 & 0.0081 \\
\hline & $\mathrm{G} / \mathrm{G}$ & 0.38 & 78 & 1.08 & 0.28 & 0.39 & 93 & 3.58 & 0.00034 & 0.38 & 171 & 3.38 & 0.00073 \\
\hline \multirow{3}{*}{ rs17820943 } & $\mathrm{T} / \mathrm{T}$ & 0.14 & 54 & -1.22 & 0.22 & 0.15 & 69 & -0.39 & 0.7 & 0.15 & 124 & -1.18 & 0.24 \\
\hline & $\mathrm{T} / \mathrm{C}$ & 0.53 & 89 & -0.53 & 0.6 & 0.49 & 127 & -2.4 & 0.017 & 0.51 & 217 & -2.1 & 0.035 \\
\hline & $\mathrm{C} / \mathrm{C}$ & 0.33 & 79 & 1.58 & 0.11 & 0.36 & 98 & 3.2 & 0.0014 & 0.35 & 177 & 3.44 & 0.00058 \\
\hline \multirow{2}{*}{ rs13041247 } & $\mathrm{C} / \mathrm{C}$ & 0.127 & 53 & -1.23 & 0.22 & 0.15 & 68 & -0.2 & 0.84 & 0.14 & 122 & -1 & 0.32 \\
\hline & $\mathrm{T} / \mathrm{T}$ & 0.332 & 77 & 0.98 & 0.33 & 0.36 & 97 & 3.17 & 0.0015 & 0.35 & 175 & 3.14 & 0.0017 \\
\hline \multirow{3}{*}{ rs11698025 } & $\mathrm{G} / \mathrm{G}$ & 0.43 & 78 & 1.5 & 0.13 & 0.47 & 94 & 2.98 & 0.0029 & 0.45 & 172 & 3.21 & 0.0013 \\
\hline & $\mathrm{G} / \mathrm{A}$ & 0.47 & 84 & -0.87 & 0.38 & 0.44 & 109 & -2.2 & 0.028 & 0.45 & 193 & -2.23 & 0.026 \\
\hline & $\mathrm{A} / \mathrm{A}$ & 0.1 & 39 & -0.81 & 0.42 & 0.085 & 50 & -0.7 & 0.48 & 0.093 & 89 & -1.061 & 0.29 \\
\hline \multirow{3}{*}{ rs6102085 } & $A / A$ & 0.2 & 57 & 0.071 & 0.94 & 0.17 & 67 & -0.93 & 0.35 & 0.18 & 125 & -0.72 & 0.47 \\
\hline & $A / G$ & 0.51 & 94 & 0.21 & 0.84 & 0.49 & 118 & -0.92 & 0.36 & 0.5 & 213 & -0.48 & 0.63 \\
\hline & $\mathrm{G} / \mathrm{G}$ & 0.29 & 68 & -0.32 & 0.75 & 0.34 & 91 & 1.89 & 0.059 & 0.32 & 159 & 1.22 & 0.22 \\
\hline
\end{tabular}

NSCLO - non-syndromic cleft lip only; NSCLP - non-syndromic cleft lip with cleft palate; NSCL/P - non-syndromic cleft lip with or without cleft palate (NSCLO and NSCLP); afreq - allele frequency; fam\# - informative family; z - vector of the large sample z statistic; bold characters indicate the items with $p$-value less than 0.0028 ( $p$-value after Bonferroni correction). 


\section{Lactate dehydrogenase and haplotype analysis}

Pair-wise lactate dehydrogenase (LD) between these SNPs was calculated by D' and $\mathrm{r}^{2}$ statistics in Haploview program (Cambridge, USA), and the results showed that rs6072081 and rs6065259 were in high LD (D'>0.96 and $\left.\mathrm{r}^{2}>0.80\right)$ across the 3 subgroups: NSCLO, NSCLP and NSCL/P (Fig. 1). To test if these 2 SNPs and other adjacent SNPs traveled together with each other from the parents to the affected child, we performed the sliding window haplotype analysis; the results indicated that A-G for rs6072081-rs6065259, A-G-C for rs6072081-rs6065259rs17820943 and A-G-C-T for rs6072081-rs6065259rs17820943-rs13041247 were statistically over-transmitted for NSCLP and NSCL/P (lowest $\mathrm{z}=3.12$ and $\mathrm{p}=0.0018$ ) (Table 5).

\section{Discussion}

NSCL/P is complex congenital craniofacial defect with serious malformations, including malocclusion, oral and nasal dysfunction. Though there has been great progress made in surgical repair, these malformations still have negative effects on the patients and their families. The past few years saw great advances in the investigation of the pathogenesis of NSCL/P. Since NSCL/P is heterogeneous, screening for the new implications in diverse ethnical populations is critical for providing more evidence in the etiology of NSCL/P. Till now, many putative candidate genes for NSCL/P had been identified successfully by GWAS and other studies. However, because of various genetic backgrounds and limitations of samples, replication studies on those candidate loci/genes among different populations were still inconsistent. ${ }^{1}$

Beaty et al. were the first to report that the $M A F B$ gene was associated with NSCL/P and participated in the craniofacial development by performing a GWAS study with the samples originated from Asian and European populations. ${ }^{12}$ To check if this gene is causal for NSCL/P in Western Han Chinese population, we selected 6 SNPs (rs6072081, rs6065259, rs17820943, rs13041247, rs11698025 and rs6102085) and replicated them among 298 case-parents trios. Interestingly, we found that rs 17820943 $\left(\mathrm{p}=0.0023 ; \mathrm{OR}_{\text {transmission }}=0.7\right.$ and $\left.95 \% \mathrm{CI}: 0.55-0.88\right)$ and $\mathrm{rs} 13041247\left(\mathrm{p}=0.0023, \mathrm{OR}_{\text {transmission }}=0.7\right.$ and $95 \%$ CI: $0.55-0.88)$ were significantly associated with the NSCL/P (Table 3). Genotypic TDT analysis further confirmed this; C/C homozygote at rs17820943 ( $\mathrm{z}=3.44$ and $\mathrm{p}=0.00058)$ and $\mathrm{T} / \mathrm{T}$ homozygote at $\mathrm{rs} 13041247(\mathrm{z}=3.14$ and $\mathrm{p}=0.0017$ ) were over-transmitted among NSCL/P trios, which indicated they could increase the risk of having an affected baby. Sliding window haplotype analysis showed haplotypes consisting of C allele at rs17820943 and $\mathrm{T}$ allele at rs13041247 were still over-transmitted among NSCL/P trios (lowest $\mathrm{p}=0.0021$ ).

To test if those 6 SNPs were independent from each other, we performed pair-wise LD analyses. The results showed strong LD between rs6072081 and rs6065259 (D'> 0.96 and $\left.\mathrm{r}^{2}>0.80\right)$ across the 3 subgroups: NSCLO, NSCLP and NSCL/P (Fig. 1); except allelic TDT analysis, genotypic

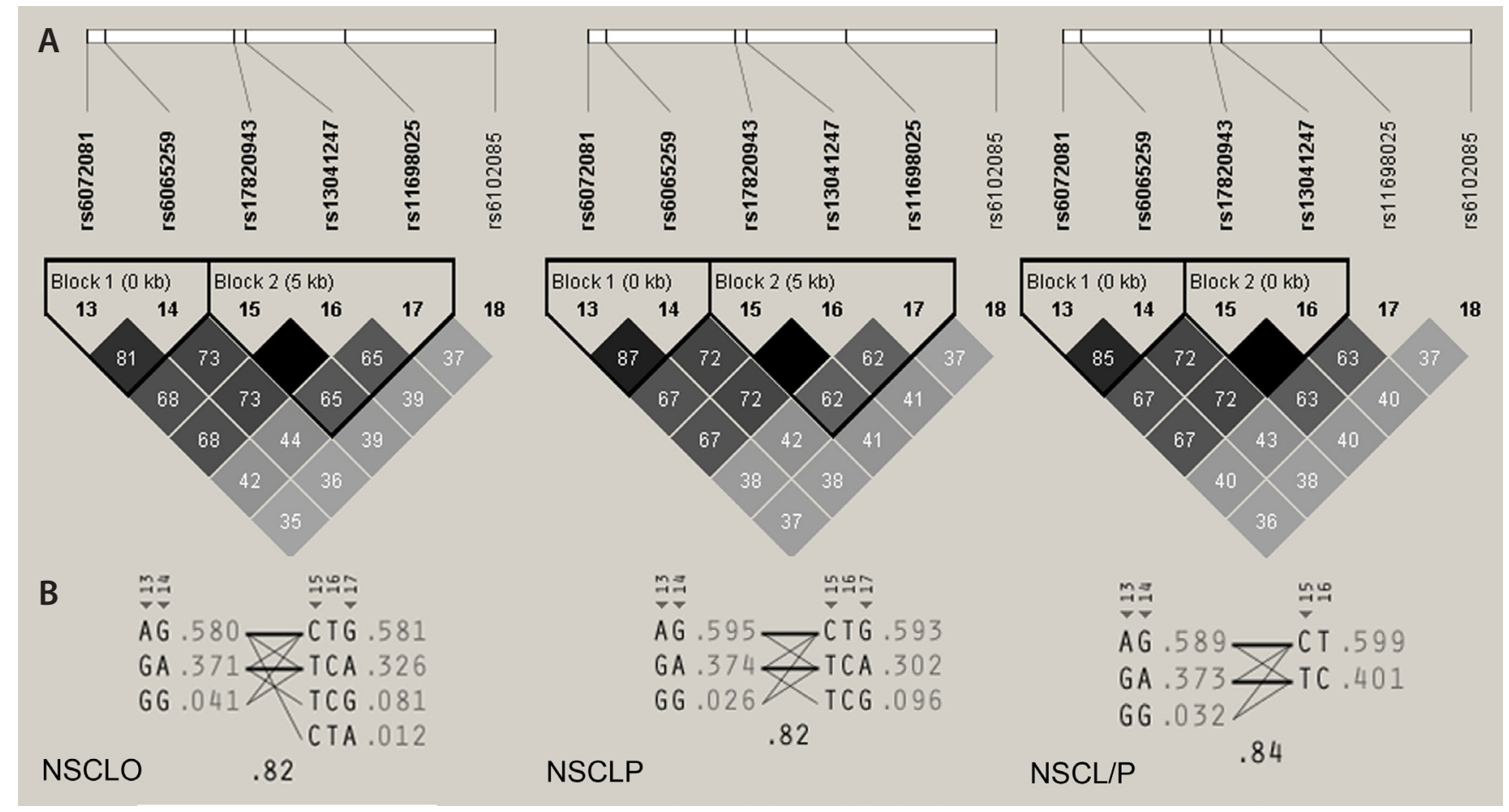

Fig. 1. Linkage disequilibrium blocks for the MAFB haplotype analysis 
Table 5. Association between the common MAFB haplotypes of single-nucleotide polymorphisms (SNPs) and non-syndromic cleft lip with or without cleft palate (NSCL/P)

\begin{tabular}{|c|c|c|c|c|c|c|c|c|c|c|}
\hline SNPs & rs6072081 & rs6065259 & rs17820943 & rs13041247 & rs11698025 & rs6102085 & afreq & fam\# & z & $\mathrm{p}$-value \\
\hline \multicolumn{11}{|c|}{ Over-transmitted } \\
\hline \multirow{2}{*}{ NSCLO } & - & - & - & T & G & A & 0.11 & 43.1 & 2.29 & 0.022 \\
\hline & - & - & C & T & G & A & 0.11 & 43.1 & 2.29 & 0.022 \\
\hline \multirow{8}{*}{ NSCLP } & A & G & - & - & - & - & 0.59 & 107 & 2.83 & 0.0047 \\
\hline & - & G & C & - & - & - & 0.57 & 109.9 & 2.36 & 0.018 \\
\hline & - & - & C & T & - & - & 0.60 & 109 & 2.27 & 0.023 \\
\hline & - & - & - & T & G & - & 0.59 & 108.9 & 2.096 & 0.036 \\
\hline & A & G & C & - & - & - & 0.56 & 109 & 2.43 & 0.015 \\
\hline & - & G & C & T & - & - & 0.57 & 107.9 & 2.37 & 0.018 \\
\hline & - & - & C & $\mathrm{T}$ & G & - & 0.59 & 108.9 & 2.072 & 0.038 \\
\hline & A & G & C & T & - & - & 0.56 & 108 & 2.43 & 0.015 \\
\hline \multirow{9}{*}{ NSCL/P } & A & G & - & - & - & - & 0.59 & 180 & 3.12 & 0.0018 \\
\hline & - & G & C & - & - & - & 0.58 & 180 & 2.66 & 0.0079 \\
\hline & - & - & C & T & - & - & 0.60 & 175 & 2.77 & 0.0056 \\
\hline & - & - & - & T & G & - & 0.59 & 179.9 & 2.55 & 0.011 \\
\hline & A & G & C & - & - & - & 0.56 & 179 & 3.07 & 0.0021 \\
\hline & - & G & C & T & - & - & 0.58 & 178 & 2.66 & 0.0079 \\
\hline & - & - & C & T & G & - & 0.59 & 178.9 & 2.53 & 0.011 \\
\hline & A & G & C & T & & - & 0.56 & 178 & 3.06 & 0.0022 \\
\hline & - & G & C & T & G & - & 0.57 & 172.9 & 2.16 & 0.031 \\
\hline \multicolumn{11}{|c|}{ Under-transmitted } \\
\hline \multirow{3}{*}{ NSCLO } & G & G & - & - & - & - & 0.04 & 15 & -1.99 & 0.047 \\
\hline & G & G & C & - & - & - & 0.025 & 10 & -2.10 & 0.036 \\
\hline & G & G & C & T & - & - & 0.025 & 10 & -2.10 & 0.036 \\
\hline \multirow{12}{*}{ NSCLP } & G & A & - & - & - & - & 0.38 & 101 & -2.48 & 0.013 \\
\hline & - & A & T & - & - & - & 0.36 & 98 & -2.06 & 0.04 \\
\hline & - & - & $T$ & C & - & - & 0.40 & 109 & -2.27 & 0.023 \\
\hline & - & - & - & C & A & - & 0.30 & 97.9 & -2.62 & 0.0088 \\
\hline & - & - & - & - & A & A & 0.27 & 81.3 & -2.27 & 0.022 \\
\hline & G & A & T & - & - & - & 0.35 & 95 & -2.01 & 0.045 \\
\hline & - & A & $T$ & C & - & - & 0.36 & 98 & -2.06 & 0.04 \\
\hline & - & - & T & C & A & - & 0.30 & 97.9 & -2.62 & 0.0088 \\
\hline & - & - & - & C & A & A & 0.26 & 79.4 & -2.33 & 0.02 \\
\hline & G & A & T & C & - & - & 0.35 & 95 & -2.01 & 0.045 \\
\hline & - & A & T & C & A & - & 0.26 & 82 & -2.26 & 0.024 \\
\hline & - & - & $\mathrm{T}$ & C & A & A & 0.26 & 79.4 & -2.33 & 0.020 \\
\hline \multirow{10}{*}{ NSCL/P } & G & A & - & - & - & - & 0.37 & 172.9 & -2.23 & 0.026 \\
\hline & G & G & - & - & - & - & 0.031 & 30 & -2.11 & 0.035 \\
\hline & - & A & T & - & - & - & 0.35 & 167.9 & -2.08 & 0.037 \\
\hline & - & - & T & C & - & - & 0.4 & 175 & -2.77 & 0.0056 \\
\hline & - & - & - & C & A & - & 0.31 & 162.7 & -2.88 & 0.004 \\
\hline & - & - & - & - & A & A & 0.27 & 150.8 & -2.27 & 0.023 \\
\hline & - & A & T & C & - & - & 0.35 & 167.9 & -2.08 & 0.037 \\
\hline & - & - & $\mathrm{T}$ & C & A & - & 0.31 & 162.7 & -2.88 & 0.004 \\
\hline & - & - & - & $C$ & A & A & 0.27 & 146.9 & -2.42 & 0.015 \\
\hline & - & - & T & C & A & A & 0.27 & 146.9 & -2.42 & 0.015 \\
\hline
\end{tabular}

NSCLO - non-syndromic cleft lip only; NSCLP - non-syndromic cleft lip with cleft palate; afreq - allele frequency; fam\# - informative family; $z$ - vector of the large sample z statistic. Listed characters indicate the items with $p$-value less than 0.05 ; bold characters indicate the items with $p$-value less than 0.0028 ( $p$-value after Bonferroni correction). 
Table 6. Parent-of-origin effect analysis by cleft types

\begin{tabular}{|c|c|c|c|c|c|c|c|c|}
\hline \multirow{2}{*}{ Cleft groups } & \multirow{2}{*}{ SNP } & \multirow{2}{*}{$\mathrm{A} 1$} & \multicolumn{2}{|c|}{ Paternal } & \multicolumn{2}{|c|}{ Maternal } & \multirow{2}{*}{ z } & \multirow{2}{*}{ p-value } \\
\hline & & & $\mathrm{T} / \mathrm{U}$ & $p$-value & $\mathrm{T} / \mathrm{U}$ & $p$-value & & \\
\hline \multirow{6}{*}{ NSCLO } & rs6072081 & G & $30.5 / 37.5$ & 0.4 & $31.5 / 37.5$ & 0.47 & -0.09 & 0.86 \\
\hline & rs6065259 & A & $26.5 / 30.5$ & 0.6 & $30.5 / 31.5$ & 0.9 & -0.29 & 0.95 \\
\hline & rs17820943 & $\mathrm{T}$ & $28.5 / 37.5$ & 0.27 & $27.5 / 38.5$ & 0.18 & 0.18 & 0.66 \\
\hline & rs13041247 & C & $28.5 / 37.5$ & 0.27 & $27.5 / 38.5$ & 0.18 & 0.18 & 0.86 \\
\hline & rs11698025 & A & $23.5 / 29.5$ & 0.41 & $26.5 / 32.5$ & 0.43 & -0.06 & - \\
\hline & rs6102085 & A & $31 / 30$ & 0.9 & $34 / 28$ & 0.45 & -0.45 & 0.24 \\
\hline \multirow{6}{*}{ NSCLP } & rs6072081 & G & $32 / 46$ & 0.11 & $26 / 48$ & 0.01 & 0.75 & 0.36 \\
\hline & rs6065259 & A & $29.5 / 44.5$ & 0.08 & $23.5 / 44.5$ & 0.011 & 0.65 & 0.63 \\
\hline & rs17820943 & $\mathrm{T}$ & $35.5 / 46.5$ & 0.22 & $27.5 / 48.5$ & 0.016 & 0.91 & 0.67 \\
\hline & rs13041247 & C & $35.5 / 46.5$ & 0.22 & $27.5 / 48.5$ & 0.016 & 0.91 & 0.36 \\
\hline & rs11698025 & $A$ & $23 / 41$ & 0.024 & $28 / 42$ & 0.09 & -0.48 & 0.63 \\
\hline & rs6102085 & A & $32.5 / 41.5$ & 0.3 & $29.5 / 43.5$ & 0.1 & 0.43 & 0.67 \\
\hline \multirow{6}{*}{$\mathrm{NSCL} / \mathrm{P}$} & rs6072081 & G & $62.5 / 83.5$ & 0.08 & $57.5 / 85.5$ & 0.019 & 0.45 & 0.65 \\
\hline & rs6065259 & $A$ & $56 / 75$ & 0.1 & $54 / 76$ & 0.054 & 0.2 & 0.84 \\
\hline & rs17820943 & $\mathrm{T}$ & $64 / 84$ & 0.1 & $55 / 87$ & 0.007 & 0.78 & 0.44 \\
\hline & rs13041247 & C & $64 / 84$ & 0.1 & $55 / 87$ & 0.007 & 0.78 & 0.44 \\
\hline & rs11698025 & $A$ & $46.5 / 70.5$ & 0.027 & $54.5 / 74.5$ & 0.08 & -0.4 & 0.69 \\
\hline & rs6102085 & $A$ & $63.5 / 71.5$ & 0.49 & $63.5 / 71.5$ & 0.49 & 0 & 1 \\
\hline
\end{tabular}

NSCLO - non-syndromic cleft lip only; NSCLP - non-syndromic cleft lip with cleft palate; NSCL/P -non-syndromic cleft lip with or without cleft palate (NSCLO and NSCLP); SNP - single nucleotide polymorphism; T/U - transmitted/non-transmitted; z - vector of the large sample z statistic.

TDT and sliding window haplotype analysis both showed that these 2 SNPs were associated with NSCL/P after multiple corrections.

Imprinting effects are increasingly regarded as a foremost source of modifiers in the complex disease. In this study, we performed the parent-of-origin effect analysis with our family-based study design. However, the results showed no significant difference between the maternal and paternal transmission (Table 6).

These 6 SNPs, all located in intergenic regions near $M A F B$, had been observed to be significantly associated with NSCL/P. ${ }^{12}$ The intergenic region did not influence the protein structure, but it exerted a potential impact on gene expression; the literature has demonstrated that DNA methylation level was higher in intergenic regions than that in the transcription start site, and it appeared more likely to be inherited. Meanwhile, DNA methylation had been found to be closely related to the alterations in chromatin structure, gene expression and disease. ${ }^{28}$

In summary, this study showed that rs17820943 and rs13041247 at MAFB gene were related with NSCL/P from Western Han Chinese, which provides new additional evidence for the pathogenesis of NSCL/P and gives new insights for future research and genetic counseling.

\section{References}

1. Mossey PA, Little J, Munger RG, Dixon MJ, Shaw WC. Cleft lip and palate. Lancet. 2009;374:1773-1785.

2. Dixon MJ, Marazita ML, Beaty TH, Murray JC. Cleft lip and palate: Understanding genetic and environmental influences. Nat Rev Genet. 2011;12:167-178.

3. Dai L, Zhu J, Mao M, et al. Time trends in oral clefts in Chinese newborns: Data from the Chinese National Birth Defects Monitoring Network. Birth Defects Res A Clin Mol Teratol. 2010;88:41-47.

4. Harville EW, Wilcox AJ, Lie RT, Vindenes H, Åbyholm F. Cleft lip and palate versus cleft lip only: Are they distinct defects? Am JEpidemiol. 2005;162:448-453.

5. Rahimov F, Marazita ML, Visel A, et al. Disruption of an AP-2alpha binding site in an IRF6 enhancer is associated with cleft lip. Nat Genet. 2008;40:1341-1347.

6. Murray JC. Gene/environment causes of cleft lip and/or palate. Clin Genet. 2002;61:248-256.

7. Xu H, Mathew G, George V. Family-based genome-wide association study for simulated data of Framingham Heart Study. BMC Proc. 2009;3(Suppl 7):S124-S128.

8. Leslie EJ, Taub MA, Liu H, et al. Identification of functional variants for cleft lip with or without cleft palate in or near PAX7, FGFR2 and NOG by targeted sequencing of GWAS Loci. Am J Hum Genet. 2015;96: 397-411.

9. Birnbaum S, Ludwig KU, Reutter $\mathrm{H}$, et al. Key susceptibility locus for nonsyndromic cleft lip with or without cleft palate on chromosome 8q24. Nat Genet. 2009;41:473-477.

10. Grant SA, Wang K, Zhang H, et al. A genome-wide association study identifies a locus for nonsyndromic cleft lip with or without cleft palate on 8q24. J Pediatr. 2009;155:909-913.

11. Mangold E, Ludwig KU, Birnbaum S, et al. Genome-wide association study identifies two susceptibility loci for nonsyndromic cleft lip with or without cleft palate. Nat Genet. 2009;42:24-26.

12. Beaty TH, Murray JC, Marazita ML, et al. A genome-wide association study of cleft lip with and without cleft palate identifies risk variants near MAFB and ABCA4. Nat Genet. 2010;42:525-529. 
13. Ludwig KU, Mangold E, Herms S, et al. Genome-wide meta-analyses of nonsyndromic cleft lip with or without cleft palate identify six new risk loci. Nat Genet. 2012;44:968-971.

14. Sun $Y$, Huang $Y$, Yin $A$, et al. Genome-wide association study identifies a new susceptibility locus for cleft lip with or without a cleft palate. Nat Commun. 2015;6:6414:doi:10.1038/ncomms7414

15. Leslie EJ, Carlson JC, Shaffer JR, et al. A multi-ethnic genome-wide association study identifies novel loci for non-syndromic cleft lip with or without cleft palate on 2p24.2, 17q23 and 19q13. Hum Mol Genet. 2016;25(13):2862-2872.

16. Marchini J, Cardon LR, Phillips MS, Donnelly P. The effects of human population structure on large genetic association studies. Nat Genet. 2004;36:512-517.

17. Li Q, Kim Y, Suktitipat B, et al. Gene-gene interaction among WNT genes for oral cleft in trios. Genet Epidemiol. 2015;39:385-394.

18. $\mathrm{XuH}$, Shete $\mathrm{S}$. Effects of population structure on genetic association studies. BMC Genet. 2005;6(Suppl 1):S109-S113.

19. Yang Y, Cvekl A. Large Maf transcription factors: Cousins of AP-1 proteins and important regulators of cellular differentiation. Einstein J Biol Med. 2007;23:2-11.

20. Pan Y, Zhang W, Du Y, et al. Different roles of two novel susceptibility loci for nonsyndromic orofacial clefts in a Chinese Han population. Am J Med Genet A. 2011;155A:2180-2185.
21. Yuan $\mathrm{QP}$, Blanton $\mathrm{SH}$, Hecht JT. Association of $A B C A 4$ and $M A F B$ with non-syndromic cleft lip with or without cleft palate. Am J Med Genet A. 2011;155A:1469-1471.

22. Lennon CJ, Birkeland AC, Nuňez JA, et al. Association of candidate genes with nonsyndromic clefts in Honduran and Colombian populations. Laryngoscope. 2012;122:2082-2087.

23. Fontoura C, Silva RM, Granjeiro JM, Letra A. Further evidence of association of the ABCA4 gene with cleft lip/palate. Eur J Oral Sci. 2012;120: 553-557.

24. Mi N, Hao Y, Jiao X, et al. Association study of single nucleotide polymorphisms of MAFB with non-syndromic cleft lip with or without cleft palate in a population in Heilongjiang Province, Northern China. Br J Oral Maxillofac Surg. 2014;52:746-750.

25. Yin X, Ma L, Li Y, et al. Genetic variants of $20 \mathrm{q} 12$ contributed to nonsyndromic orofacial clefts susceptibility. Oral Dis. 2017;23(1):50-54.

26. Duan SJ, Huang N, Zhang BH, et al. New insights from GWAS for the cleft palate among han Chinese population. Med Oral Patol Oral Cir Bucal. 2017;22(2):e219-e227.

27. Yu Q, He S, Zeng N, et al. BMP7 Gene involved in nonsyndromic orofacial clefts in Western Han Chinese. Med Oral Patol Oral Cir Bucal. 2015:20:e298-304.

28. Rowlatt A, Hernández-Suárez G, Sanabria-Salas MC, et al. The heritability and patterns of DNA methylation in normal human colorectum. Hum Mol Genet. 2016;25(12):2600-2611. 